\title{
GAI Functions in the Plant Response to Dehydration Stress in Arabidopsis thaliana
}

\author{
Zhijuan Wang ${ }^{1, \dagger}$, Liu Liu ${ }^{2,3, \dagger}$, Chunhong Cheng ${ }^{2,3, \dagger}$, Ziyin Ren ${ }^{1}$, Shimin $\mathrm{Xu}^{1}$ and Xia Li ${ }^{1, *}$ \\ 1 State Key Laboratory of Agricultural Microbiology, College of Plant Science and Technology, Huazhong \\ Agricultural University, Wuhan 430070, China; wzhijuan@163.com (Z.W.); rzy1819345962@163.com (Z.R.); \\ 15527847403@163.com (S.X.) \\ 2 The State Key Laboratory of Plant Cell \& Chromosome Engineering, Center for Agricultural Research \\ Resources, Institute of Genetics and Developmental Biology, Chinese Academy of Sciences, 286 Huaizhong \\ Road, Shijiazhuang 050021, China; liuliu19890729@163.com (L.L.); xiaobei15109217512@163.com (C.C.) \\ 3 Graduate University of Chinese Academy of Sciences, No.19A Yuquan Road, Beijing 100049, China \\ * Correspondence: xli@mail.hzau.edu.cn; Tel.: +86-027-87856637 \\ + These authors contributed equally to this work.
}

Received: 20 December 2019; Accepted: 23 January 2020; Published: 27 January 2020

\begin{abstract}
DELLA (GAI/RGA/RGL1/RGL2/RGL3) proteins are key negative regulators in GA (gibberellin) signaling and are involved in regulating plant growth as a response to environmental stresses. It has been shown that the DELLA protein PROCERA (PRO) in tomato promotes drought tolerance, but its molecular mechanism remains unknown. Here, we showed that the gai-1 (gibberellin insensitive 1) mutant (generated from the gai-1 (Ler) allele (with a 17 amino acid deletion within the DELLA domain of GAI) by backcrossing gai-1 (Ler) with Col-0 three times), the gain-of-function mutant of GAI (GA INSENSITIVE) in Arabidopsis, increases drought tolerance. The stomatal density of the gai-1 mutant was increased but its stomatal aperture was decreased under abscisic acid (ABA) treatment conditions, suggesting that the drought tolerance of the gai-1 mutant is a complex trait. We further tested the interactions between DELLA proteins and ABF2 (abscisic acid (ABA)-responsive element (ABRE)-binding transcription factors) and found that there was a strong interaction between DELLA proteins and ABF2. Our results provide new insight into DELLA proteins and their role in drought stress tolerance.
\end{abstract}

Keywords: drought; GA; DELLA; ABF2; protein-protein interaction

\section{Introduction}

A water deficit is a restrictive factor for plant development, productivity, and geographical distribution. Plants have evolved varied strategies to cope with decreased water availability, including promoting stomatal closure and altered plant growth and development. The stress-induced hormone abscisic acid (ABA) plays an important role in a plant's response to drought tolerance [1-3]. Increasing evidence has proven that gibberellin (GA) plays a negative role in drought response. The over-accumulation of the GA mutant or increased GA activity shows an increased water deficit sensitivity, whereas a GA-deficient mutant or decreased GA activity shows an increased water deficit tolerance [4-8].

DELLA (GAI/RGA/RGL1/RGL2/RGL3) proteins are major negative regulators of GA signaling. In the absence of GA, DELLA proteins inhibit the GA-dependent processes, including germination, growth, and flowering. Under increased GA levels, GA binds to its nuclear receptor GID1 (GA insensitive dwarf1) and changes its conformation, leading to its interaction with the N-terminal end of DELLA proteins [9-11]. The interaction of DELLA proteins with GID1 causes its ubiquitination and subsequent 
degradation by the $26 \mathrm{~S}$ proteasome, leading to the activation of GA responses [12,13]. DELLA proteins are involved in most GA-mediated plant growth and environmental stresses, including dehydration stress. Recently, it was reported that the DELLA protein PRO (PROCERA) in tomato functions positively in the plant response to drought stress. The loss-of-function of the PRO mutant shows a reduced tolerance to drought, whereas the overexpression of the constitutively active stable $P R O$ increases drought tolerance [8]. However, the molecular mechanism of DELLA proteins remains unclear.

There are five DELLA members in Arabidopsis: GAI (GA INSENSITIVE), RGA (REPRESSOR OR GAI3), RGL1 (RGA-LIKE1), RGL2, and RGL3. To uncover the molecular mechanism that determines how DELLA proteins function in drought tolerance, we used GAI as a sample to analyze the function of DELLA proteins in response to drought stress. We made use of a gain-of-function mutant gai-1 generated from the gai-1 (Ler) allele (with a 17 amino acid deletion within the DELLA domain of GAI) by backcrossing gai-1 (Ler) with Col-0 (Columbia-0) three times. We showed that this mutant has an increased drought tolerance phenotype. Further, we found that GAI and other DELLA proteins interacted with ABF2 (abscisic acid (ABA)-responsive element (ABRE)-binding transcription factors), the transcriptional factor that plays a pivotal role in ABA signaling for drought tolerance. Our results thus shed some light on the mechanism behind how DELLA proteins function in drought stress tolerance.

\section{Results}

To study the function of DELLA proteins in drought tolerance in Arabidopsis, we firstly analyzed the phenotype of the gai-1 mutant under the condition of drought treatment. Three-week-old seedlings of wild type (Col-0) and mutant gai-1 were withheld from water for 21 days. The wild type plants were severely wilted, whereas the gai-1 mutant did not wilt and continued to grow. After rewatering, all of the gai-1 plants recovered, whereas none of the wild type plants survived (Figure 1A), indicating that the gai-1 mutant is more tolerant to drought and that GAI is a positive regulator in the plant response to drought tolerance. The function of GAI in drought tolerance is consistent with that of PRO in tomato, suggesting that this tolerance is a conserved function of DELLA proteins in the plant kingdom.

Changes in transpiration rate could account for the altered tolerance to drought. We then tested the water loss rate of the detached leaves. Leaves of 3 week old seedlings were cut and exposed to air and were weighted at regular time points. To our surprise, the gai-1 mutant leaves lost their water at a much higher rate than the wild type leaves (Figure 1B). The water loss of the wild type sample was only $20 \%$, whereas the water loss of the gai-1 mutant was over $30 \%$ at $4 \mathrm{~h}$ after exposure to air, suggesting that the gai-1 mutant is sensitive to dehydration when detached leaves are exposed to air.

The stomata are key channels that control gas exchange and water evaporation. We then tested the stomatal density and aperture from leaves of wild type and gai-1 plants grown in soil. The stomatal density of the gai-1 mutant was significantly higher than that of the wild type plant $(2.6 \times)$ (Figure 1C,D). This may be the reason for the higher rate water loss in the gai-1 mutant for the detached leaves. For the stomatal apertures, the wild type and gai-1 mutant were comparable under $\mathrm{KCl}$-treated control conditions. However, under ABA treatment, the stomatal aperture of the gai-1 mutant was much smaller than that of the wild type (Figure 1E,F). The stomatal density and aperture of the gai-1 mutant under stress conditions are consistent with those of the PRO gain-of-function mutant, suggesting that this is a conserved mechanism for DELLA proteins in regulating plant development and environmental adaption.

Generally, DELLA proteins function by interacting with other transcriptional factors. As ABF2 (abscisic acid (ABA)-responsive element (ABRE)-binding transcription factors) is a key regulator in drought tolerance, we hypothesized that GAI interacts with $\mathrm{ABF} 2$ to increase drought tolerance. To test this hypothesis, we tested the interaction between GAI and ABF2 via yeast two-hybrid and BiFC (bimolecular fluorescent complimentary) assays. In the yeast two-hybrid assay, GAI and ABF2 were recombined to the gateway-compatible destination vectors pGADT7-DEST (AD) and PGBKT7-DEST (BD), respectively. The AD and BD constructs were cotransformed to the yeast strain AH109, and 
their interaction was determined by the growth on the SD (synthetic dropout) medium lacking Trp (tryptophan), Leu (leucine), His (histidine), and Ade (adenine). There was a strong interaction between GAI and ABF2 in the yeast two-hybrid assay (Figure 2A). For the BiFC assay, GAI and ABF2 were recombined to pEarleyGate201-YN (N-terminal YFP (yellow fluorescent protein)) and pEarleyGate202-YC (C-terminal YFP), respectively. Both constructs were coinfiltrated into Nicotiana benthamiana leaves. The YFP signal was observed in the nucleus of the plant cell coexpressing GAI-YFP and ABF2-YFPC (Figure 2B), but no YFP signal was detected in the plant cell coexpressing GAI-YFPN and empty YFPC or ABF2-YFPC and empty YFPN (Figure S1), indicating that GAI and ABF2 interacted in the nucleus of the plant cell. We also tested the interactions between other DELLA proteins, including RGA, RGL1, RGL2, and RGL3, with ABF2. The yeast two-hybrid and BiFC assays both showed that all of the DELLA proteins interacted with ABF2 (Figure 2A,B).

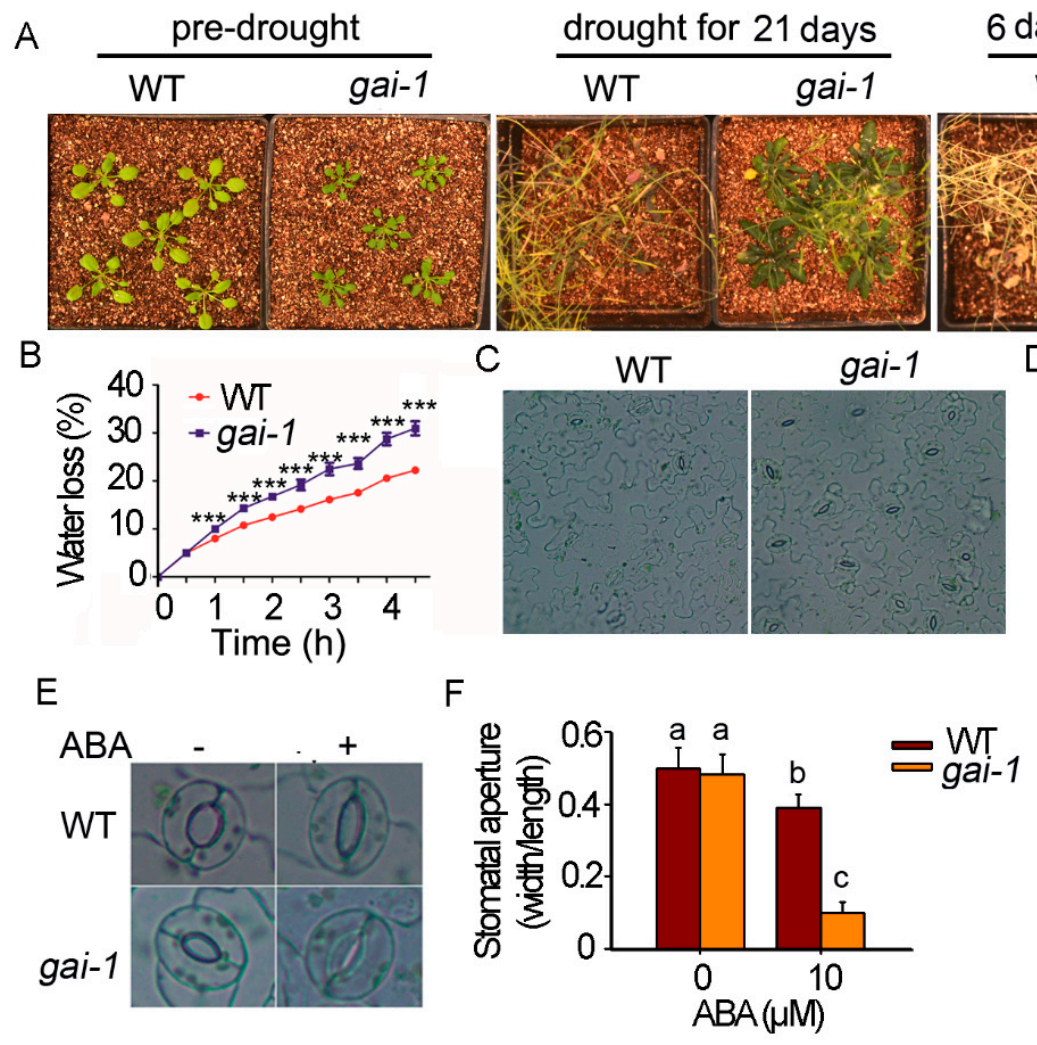

Figure 1. The gai-1 (gibberellin insensitive 1) mutant is more tolerant to drought stress than WT (wild type). (A) gai-1 mutant plants showed tolerance to dehydration stress. gai-1 mutant plants showed the ability to withstand long drought conditions without negative effects whereas the wild type under the same conditions completely wilted. (B) gai-1 plants showed increased water loss compared to WT. Data shown are the means \pm SDs from three biological repeats $(n=3$, eight leaves from eight plants were used for each repeat, $p<0.001$ ). (C,D) Stomatal density of WT and gai-1 mutant. Stomatal density was observed from comparable age leaves of 3 week old wild type and gai-1 plants. The stomatal density was represent by number of stomata per millimeters squared. Data shown are the means \pm SDs from three biological repeats ( $n=3$, five leaves from five plants were used for each repeat, $p<0.001$ ). (E) Representative stomata of the WT and gai-1 mutant under control and abscisic acid (ABA) treatment conditions. Leaves of the WT and gai-1 mutant were treated with $10 \mu \mathrm{M}$ ABA for 2 $\mathrm{h}(+)$, and (-) represents leaves without ABA treatment. (F) Stomatal apertures of the WT and gai-1 mutant corresponding to (E). Values are mean ratios of width to length \pm SDs of three independent experiments. Letters indicate significant differences from the WT (0 ABA treatment) according to the Student's Newman-Kuels test $\left({ }^{* *} p<0.05\right)$. 

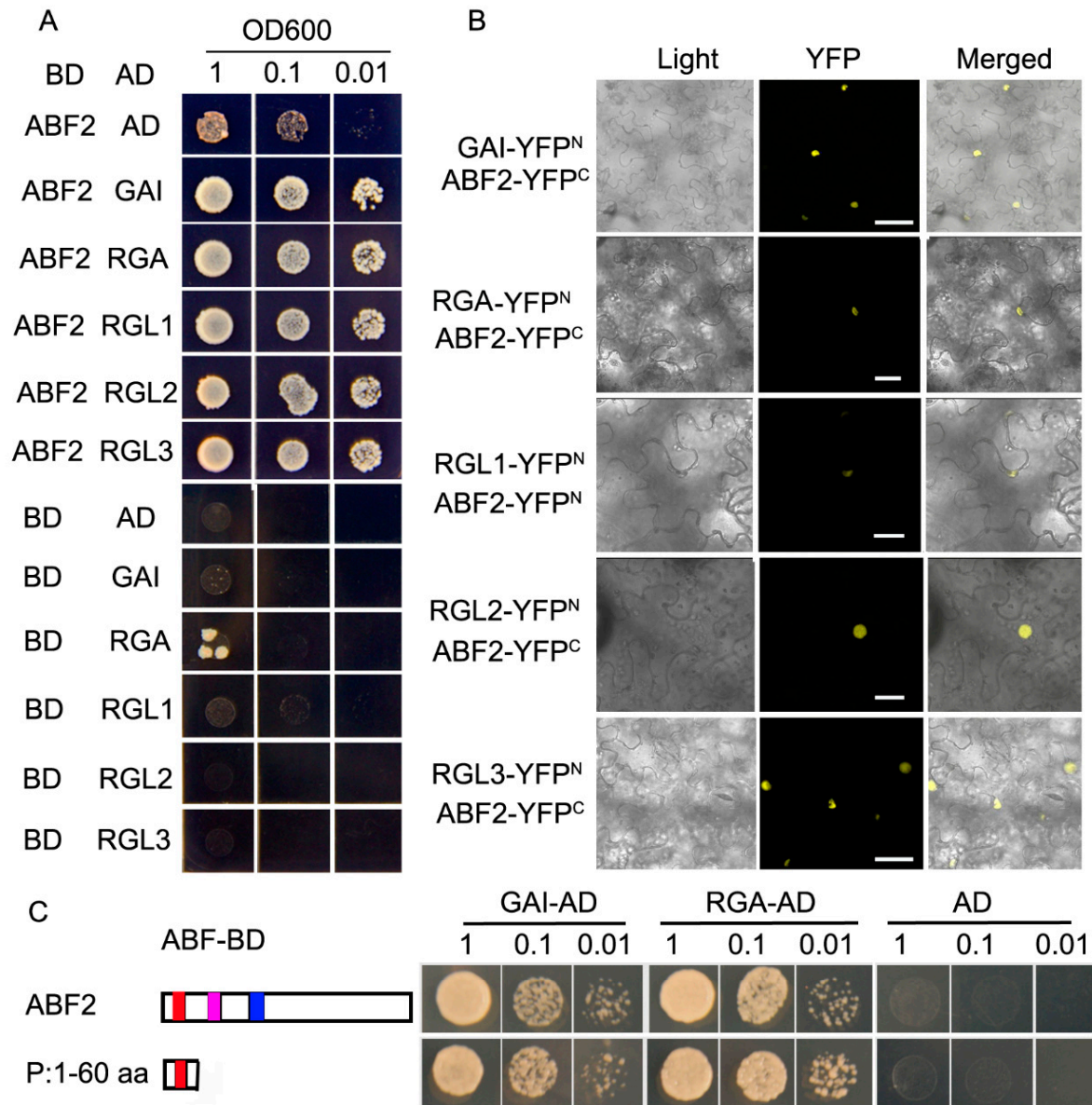

Q:61-116 aa

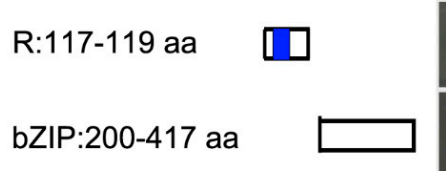

BD

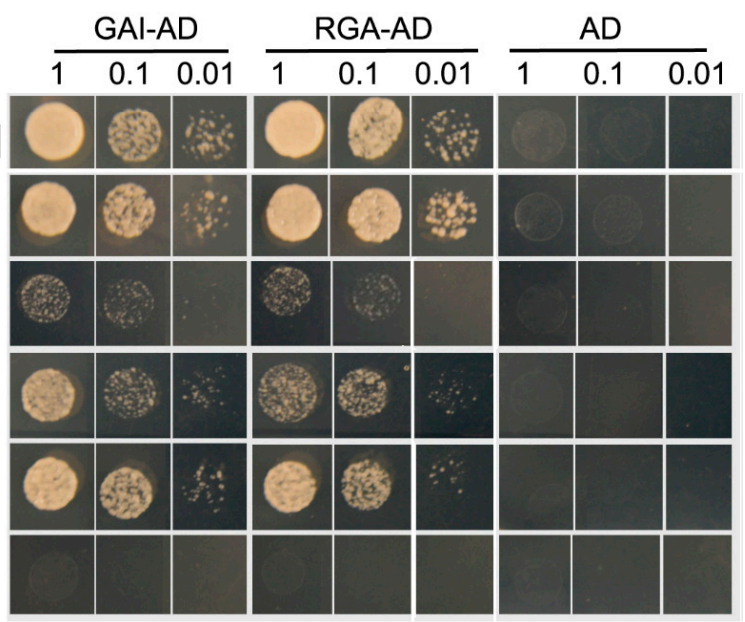

Figure 2. DELLA (GAI/RGA/RGL1/RGL2/RGL3) proteins interacted with ABF2 (abscisic acid (ABA)-responsive element (ABRE)-binding transcription factors). (A) DELLA proteins interacted with ABF2 in yeast two-hybrid assay. The yeast cells expressing the indicated constructs were spotted as a series of three dilutions. The yeast cells expressing the constructs of ABF2-pGBKT7-DEST (BD)/GAI-pGADT7-DEST (AD), ABF2-BD/RGA (REPRESSOR OR GAI3)-AD, ABF2-BD/RGL1 (RGA-LIKE1)-AD, ABF2-BD/RGL2-AD, and ABF2-BD/RGL3-AD grew better on the $\mathrm{SD}$ medium than that of yeast growth cells expressing the control's constructs. (B) BiFC (bimolecular fluorescent complimentary) assay between DELLA proteins and ABF2. Nicotiana benthamiana leaves were co-transformed with the constructs containing the indicated YFP (yellow fluorescent protein) $\mathrm{N}$-terminal $\left(\mathrm{YFP}^{\mathrm{N}}\right.$ ) and YFP C-terminal (YFPC) fusions, and YFP was imaged $48 \mathrm{~h}$ after transformation. Bars $=50 \mu \mathrm{m}$. (C) Interaction assay between GAI and RGA with ABF2 fragments. P: 1-60 amino acid; Q: 61-116 amino acid; R: 117-199 amino acid; bZIP (basic region/leucine zipper): 200-417 amino acid. The yeast cells expressing the indicated constructs were spotted as a series of three dilution. The yeast cell expressing the constructs of ABF2-BD/GAI-AD, ABF2-BD/RGA-AD, ABF2P-BD/GAI-AD, ABF2P-BD/RGA-AD, ABF2bZIP-BD/GAI-AD, ABF2bZIP-BD/RGA-AD, grew more effectively on the $\mathrm{SD}$ medium than that of yeast cells expressing ABF2R-BD/RGA-AD, ABF2R-BD/GAI-AD, ABF2Q-BD/ RGA-AD, and ABF2Q-BD/GAI-AD. 
There are three conserved motifs in the N-terminal of ABF2: $\mathrm{P}, \mathrm{Q}$, and $\mathrm{R}$. The P motif is responsible for transactivation activity and activates downstream gene expression [14]. To test which motif is responsible for interacting with GAI, we tested the interaction between GAI and the $\mathrm{P}, \mathrm{Q}, \mathrm{R}$, and bZIP (basic region/leucine zipper) motifs of ABF2. Our yeast two-hybrid assay showed that there was a strong interaction between the GAI and the P and bZIP motifs, whereas the interaction between GAI and the $\mathrm{Q}$ and $\mathrm{R}$ motifs was much weaker (Figure 2C). We also tested the interaction between RGA and the $\mathrm{P}, \mathrm{Q}, \mathrm{R}$, and bZIP motifs of ABF2. RGA also showed a strong interaction with the $\mathrm{P}$ and bZIP motifs but a weak interaction with the $Q$ and $R$ motifs (Figure $2 \mathrm{C}$ ).

\section{Discussion}

GA is an important phytohormone that regulates plant growth. Increasing evidence has demonstrated that GA plays a role in the response to environmental stresses. DELLA proteins are key negative regulators in GA signaling, and our results showed that DELLA proteins increased drought tolerance by interacting with $\mathrm{ABF} 2$, a positive regulator in the ABA signaling pathway.

The cellular mechanism of the drought tolerance of the gai-1 mutant seems complex and confusing. The stomatal density of the gai-1 mutant is higher than that of the wild type, which makes water loss occur more quickly. Indeed, we found that the water loss rate of the detached leaves in the gai-1 mutant was higher than that of the wild type (Figure 1B). The stomatal aperture in ABA treatment was found to be smaller in the gai-1 mutant than that of the wild type sample, which may be responsible for the drought tolerance. The stomata's phenotype, density, and aperture of the gai-1 mutant are similar to those of the gain-of-function of PRO in tomato [8], suggesting that it is conserved for DELLA proteins in regulating plant development and stress response.

As DELLA proteins do not have a DNA binding domain, it is common for them to interact with other transcription factors to regulate downstream target genes. For example, DELLA proteins interact with ABI3 and ABI5 to activate SOM (SOMNUS) expression at high temperatures [15]; RGA interacts with BZR1 (Brassinazole-resistant 1) to inhibit its transcriptional activities to downstream genes to regulate cell growth [16]; RGA interacts with WRKY6 (WRKYGQK) to block its transcriptional activities on its downstream genes, SAG13 (Senescence-associated gene13) and SGR (Stay green), to regulate senescence [17]; and RGL2 interacts with the NF-YC (NUCLEAR FACTOR-Y C) homologues NF-YC3, NF-YC4, and NF-YC9 to activate the downstream gene ABI5 to regulate seed germination [18]. Here, we showed that DELLA proteins interact with ABF2 to regulate drought tolerance. AREB/ABF (abscisic acid-responsive element binding) proteins play pivotal roles in the regulation of plant responses to abiotic stresses. By binding to the ABRE element in the promoter region of stress-responsive genes, $A R E B / A B F$ factors regulate gene expression under drought stress $[19,20]$. In Arabidopsis, four AREB/ABF factors, namely, AREB1/ABF2, AREB2/ABF4, ABF1, and ABF3, are induced by ABA and osmotic stress [21,22]. Overexpressing AREB1/ABF2, AREB2/ABF4, or ABF3 promotes drought tolerance, and a loss-of-function of these genes enhances drought sensitivity $[14,16,23,24]$. Many stress-inducible genes, including $R D 29 B$ and $R A B 18$, were downregulated in the areb1 areb2 abf3 abf1-2 quadruple mutant [20]. Our results showed a strong interaction between DELLA proteins and the $\mathrm{ABF} 2$ protein (Figure 2A,B). It is reasonable that the interaction of DELLA proteins with ABF2 could activate $\mathrm{ABF} 2$ transcriptional activity to promote drought tolerance. A further expression assay of the downstream gene in the gai-1 mutant and the binding assay of ABF2 to the promoter of $R D 29 B$ or $R A B 18$ in the presence or absence of DELLA proteins would allow for the determination of the role of the interaction between DELLA proteins and ABF2. We also cannot exclude the possibility that DELLA proteins interact with other AREB/ABF factors, such as AREB2/ABF4, ABF1, or ABF3. A further interaction assay between the DELLA proteins and other AREB/ABF factors will deepen our understanding of the role of DELLA proteins in drought tolerance.

In summary, our results showed that GAI increased drought tolerance in Arabidopsis. GAI had conserved functions in increasing the stomatal density and decreasing the stomatal aperture under ABA treatment conditions. Further, we showed that GAI interacted with ABF2, especially the 
$\mathrm{N}$-terminal end P domain and the bZIP domain of ABF2. Our results provide new insight into DELLA protein functions in drought stress tolerance and the crosstalk between ABA and GA in response to drought tolerance.

\section{Materials and Methods}

\subsection{Plant Materials and Growth Conditions}

Arabidopsis thaliana ecotype Col-0 was used as the wild type in this study. The gai-1 mutant was kindly gifted by Dr. Xiangdong Fu (Institute of Genetics and Developmental Biology, CAS). The seeds were germinated and grown on MS (Murashige \& Skoog) medium and transplanted into soil at 10 days after germination. The plants were grown under a $16 \mathrm{~h}$ light $/ 8 \mathrm{~h}$ dark photoperiod at $23^{\circ} \mathrm{C}$.

\subsection{Drought Treatment, Water Loss Analysis, and Stomatal Aperture Measurement}

For measurement of drought tolerance, water was withheld from 21 day old wild-type and gai-1 mutant plants. After 21 days of drought treatment, the plants were rewatered; the plants were photographed 6 days after re-watering. For measurement of water loss, eight rosette leaves from eight plants were detached from 3 week old well-watered plants and weighted at the indicated times. For the stomatal function, rosette leaves from well-watered plant were incubated in a solution containing $50 \mathrm{mM} \mathrm{KCl}, 10 \mathrm{mM} \mathrm{CaCl}_{2}$, and $10 \mathrm{mM} \mathrm{MES} \mathrm{(pH} \mathrm{6.15)} \mathrm{for} 2 \mathrm{~h}$ under light. ABA was then added to the solution to a final concentration of $10 \mu \mathrm{M}$. After ABA treatment for $2 \mathrm{~h}$, stomatal apertures were measured as described previously [2].

\subsection{Protein-Protein Analysis}

The constructs were created in two pairs of Gateway-compatible destination vectors: pGBKT7-DEST (BD) with pGBAD7-DEST (AD) and pEarleyGate201-YN (N-terminal YFP) with pEarleyGate202-YC (C-terminal YFP) [25]. The coding sequences of GAI, RGA, RGL1, RGL2, RGL3, $A B F 2$, and different deletion fragments of $A B F 2$ were amplified from Col- 0 cDNA, inserted into pDONR207, and then recombined in the appropriate destination vector. Yeast two-hybrid and BiFC assays were performed as previously described [2]. For Y2H, Saccharomyces cerevisiae strain AH109 was used for co-transformation of the $\mathrm{AD}$ and BD constructs. A series of $5 \mu \mathrm{L}$ aliquots of diluted co-transformed AH109 culture was spotted onto SD plates lacking Trp, Leu, His, and Ade, and incubated at $30^{\circ} \mathrm{C}$ for $2-5$ days. Plasmids pGBKT7 and pGADT7-Rec were used as negative controls. For the BiFC assay, Agrobacterium tumefaciens GV3101 carrying the YFP N-terminal and YFP C-terminal fusion constructs was infiltrated into $N$. benthamiana leaves, as described by Luo et al. [2]. The reconstituted YFP signals were observed using confocal imaging $48 \mathrm{~h}$ after infiltration. Empty vectors were used as negative controls.

Supplementary Materials: The following are available online at http:/www.mdpi.com/1422-0067/21/3/819/s1.

Author Contributions: Conceptualization, X.L.; methodology, Z.W., C.C., L.L., Z.R., and S.X.; validation, Z.W., and Z.R.; formal analysis, Z.W. and L.L; investigation, X.L.; writing-original draft preparation, Z.W.; writing-review and editing, X.L.; funding acquisition, X.L. All authors have read and agreed to the published version of the manuscript.

Funding: This research was funded by the National Key Research and Development Program of China, grant number 2016YFA0500503; Fundamental Research Funds for Central Universities, grant number 2662018PY075; and Huazhong Agricultural University's Scientific and Technological Self-innovation Foundation, grant number 2015RC014.

Acknowledgments: We thank Xiangdong Fu (Institute of Genetics and Developmental Biology, CAS) for providing gai-1 mutant seed.

Conflicts of Interest: The authors declare no conflict of interest. The funders had no role in the design of the study; in the collection, analyses, or interpretation of data; in the writing of the manuscript, or in the decision to publish the results. 


\section{Abbreviations}

$\begin{array}{ll}\text { ABA } & \text { Abscisic acid } \\ \text { AREB/ABF } & \text { Abscisic acid-responsive element binding } \\ \text { GA } & \text { Gibberellin } \\ \text { GAI } & \text { GA INSENSITIVE } \\ \text { GID1 } & \text { GA insensitive dwarf1 } \\ \text { PRO } & \text { PROCERA }\end{array}$

\section{References}

1. Munns, R. Comparative physiology of salt and water stress. Plant Cell Environ. 2002, 25, 239-250. [CrossRef] [PubMed]

2. Luo, Y.; Wang, Z.; Ji, H.; Fang, H.; Wang, S.; Tian, L.; Li, X. An Arabidopsis homolog of importin $\beta 1$ is required for ABA response and drought tolerance. Plant J. 2013, 75, 377-389. [CrossRef] [PubMed]

3. Ku, Y.S.; Sintaha, M.; Cheung, M.Y.; Lam, H.M. Plant Hormone Signaling Crosstalks between Biotic and Abiotic Stress Responses. Int. J. Mol. Sci. 2018, 19, 3206. [CrossRef] [PubMed]

4. Achard, P.; Cheng, H.; De Grauwe, L.; Decat, J.; Schoutteten, H.; Moritz, T.; Van Der Straeten, D.; Peng, J.; Harberd, N.P. Integration of plant responses to environmentally activated phytohormonal signals. Science 2006, 311, 91-104. [CrossRef]

5. Li, J.; Sima, W.; Ouyang, B.; Wang, T.; Ziaf, K.; Luo, Z.; Liu, L.; Li, H.; Chen, M.; Huang, Y.; et al. Tomato SIDREB gene restricts leaf expansion and internode methylation and chromatin patterning elongation by downregulating key genes for gibberellin biosynthesis. J. Exp. Bot. 2012, 63, 695-709. [CrossRef]

6. Colebrook, E.H.; Thomas, S.G.; Phillips, A.L.; Hedden, P. The role of gibberellin signalling in plant responses to abiotic stress. J. Exp. Biol. 2014, 217, 67-75. [CrossRef]

7. Nir, I.; Moshelion, M.; Weiss, D. The Arabidopsis gibberellin methyl transferase 1 suppresses gibberellin activity, reduces whole-plant transpiration and promotes drought tolerance in transgenic tomato. Plant Cell Environ. 2014, 37, 113-123. [CrossRef]

8. Nir, I.; Shohat, H.; Panizel, I.; Olszewski, N.; Aharoni, A.; Weiss, D. The Tomato DELLA Protein PROCERA Acts in Guard Cells to Promote Stomatal Closure. Plant Cell 2017, 29, 3186-3197. [CrossRef]

9. Harberd, N.P.; Belfield, E.; Yasumura, Y. The angiosperm gibberellin-GID1-DELLA growth regulatory mechanism: How an "inhibitor of an inhibitor" enables flexible response to fluctuating environments. Plant Cell 2009, 21, 1328-1339. [CrossRef] [PubMed]

10. Schwechheimer, C. Gibberellin signaling in plants-the extended version. Front. Plant Sci. 2012, 2, 107. [CrossRef] [PubMed]

11. Sun, T.P. The molecular mechanism and evolution of the GA-GID1-DELLA signaling module in plants. Curr. Biol. 2011, 21, R338-R345. [CrossRef] [PubMed]

12. Gao, X.H.; Xiao, S.L.; Yao, Q.F.; Wang, Y.J.; Fu, X.D. An updated GA signaling 'relief of repression' regulatory model. Mol. Plant 2011, 4, 601-606. [CrossRef] [PubMed]

13. Daviere, J.M.; Achard, P. A pivotal role of DELLAs in regulating multiple hormone signals. Mol. Plant 2016, 9, 10-20. [CrossRef] [PubMed]

14. Fujita, Y.; Fujita, M.; Satoh, R.; Maruyama, K.; Parvez, M.M.; Seki, M.; Hiratsu, K.; Ohme-Takagi, M.; Shinozaki, K.; Yamaguchi-Shinozaki, K. AREB1 is a transcription activator of novel ABRE-dependent ABA signaling that enhances drought stress tolerance in Arabidopsis. Plant Cell 2005, 17, 3470-3488. [CrossRef] [PubMed]

15. Lim, S.; Park, J.; Lee, N.; Jeong, J.; Toh, S.; Watanabe, A.; Kim, J.; Kang, H.; Kim, D.H.; Kawakami, N.; et al. ABA-insensitive3, ABA-insensitive5, and DELLAs Interact to activate the expression of SOMNUS and other high-temperature-inducible genes in imbibed seeds in Arabidopsis. Plant Cell 2013, 25, 4863-4878. [CrossRef]

16. Li, Q.F.; Wang, C.; Jiang, L.; Li, S.; Sun, S.S.; He, J.X. An interaction between BZR1 and DELLAs mediates direct signaling crosstalk between brassinosteroids and gibberellins in Arabidopsis. Sci. Signal. 2012, 5, ra72. [CrossRef]

17. Zhang, Y.; Liu, Z.; Wang, X.; Wang, J.; Fan, K.; Li, Z.; Lin, W. DELLA proteins negatively regulate dark-induced senescence and chlorophyll degradation in Arabidopsis through interaction with the transcription factor WRKY6. Plant Cell Rep. 2018, 37, 981-992. [CrossRef] 
18. Liu, X.; Hu, P.; Huang, M.; Tang, Y.; Li, Y.; Li, L.; Hou, X. The NF-YC-RGL2 module integrates GA and ABA signalling to regulate seed germination in Arabidopsis. Nat. Commun. 2016, 7, 12768. [CrossRef]

19. Nakashima, K.; Ito, Y.; Yamaguchi-Shinozaki, K. Transcriptional regulatory networks in response to abiotic stresses in Arabidopsis and grasses. Plant Physiol. 2009, 149, 88-95. [CrossRef]

20. Yoshida, T.; Fujita, Y.; Maruyama, K.; Mogami, J.; Todaka, D.; Shinozaki, K.; Yamaguchi-Shinozaki, K. Four Arabidopsis AREB/ABF transcription factors function predominantly in gene expression downstream of SnRK2 kinases in abscisic acid signaling in response to osmotic stress. Plant Cell Environ. 2015, 38, 35-49. [CrossRef]

21. Fujita, Y.; Fujita, M.; Shinozaki, K.; Yamaguchi-Shinozaki, K. ABA mediated transcriptional regulation in response to osmotic stress in plants. J. Plant Res. 2011, 124, 509-525. [CrossRef] [PubMed]

22. Chen, K.; Li, G.; Bressan, R.A.; Song, C.; Zhu, J.; Zhao, Y. Abscisic acid dynamics, signaling and functions in plants. J. Integr. Plant Biol. 2020, 62, 25-54. [CrossRef] [PubMed]

23. Yoshida, T.; Fujita, Y.; Sayama, H.; Kidokoro, S.; Maruyama, K.; Mizoi, J.; Shinozaki, K.; Yamaguchi-Shinozaki, K. AREB1, AREB2, and ABF3 are master transcription factors that cooperatively regulate ABRE-dependent ABA signaling involved in drought stress tolerance and require ABA for full activation. Plant J. 2010, 61, 672-685. [CrossRef] [PubMed]

24. Singh, D.; Laxmi, A. Transcriptional regulation of drought response: A tortuous network of transcriptional factors. Front. Plant Sci. 2015, 6, 895. [CrossRef]

25. Lu, Q.; Tang, X.; Tian, G.; Wang, F.; Liu, K.; Nguyen, V.; Kohalmi, S.E.; Keller, W.A.; Tsang, E.W.; Harada, J.J.; et al. Arabidopsis homolog of the yeast TREX2 mRNA export complex: Components and anchoring nucleoporin. Plant J. 2010, 61, 259-270. [CrossRef]

(C) 2020 by the authors. Licensee MDPI, Basel, Switzerland. This article is an open access article distributed under the terms and conditions of the Creative Commons Attribution (CC BY) license (http://creativecommons.org/licenses/by/4.0/). 